Western University Scholarship@Western

Electrical and Computer Engineering Publications Electrical and Computer Engineering Department

$12-2003$

\title{
UML Extensions for Real-Time Control Systems
}

Qimin Gao

Dematic, gaoqimin@hotmail.com

Lyndon Brown

Western University, lbrown@eng.uwo.ca

Luiz Fernando Capretz

Western University, lcapretz@uwo.ca

Follow this and additional works at: https://ir.lib.uwo.ca/electricalpub

Part of the Computer Engineering Commons, and the Electrical and Computer Engineering Commons

\section{Citation of this paper:}

Gao, Qimin; Brown, Lyndon; and Capretz, Luiz Fernando, "UML Extensions for Real-Time Control Systems" (2003). Electrical and Computer Engineering Publications. 80.

https://ir.lib.uwo.ca/electricalpub/80 
$42^{\text {nd }}$ IEEE Conference on Decision and Control, Maui, Hawaii, pp. 5932-5938, December 2003

\title{
UML Extensions for Real-Time Control Systems
}

\author{
Qimin Gao L.J.Brown $\quad$ L.F.Capretz \\ Department of Electrical and Computer Engineering \\ The University of Western Ontario \\ London, Ontario, Canada N6A 5B9
}

February 25, 2003

\begin{abstract}
The use of object oriented techniques and methodologies for the design of real-time control systems appears to be necessary in order to deal with the increasing complexity of such systems. Recently many object-oriented methods have been used for the modeling and design of real-time control systems. We believe that an approach that integrates the advancements in both object modeling and design methods, and real-time scheduling theory is the key to successful use of object oriented technology for real-time software. Surprisingly several past approaches to integrate the two either restrict the object models, or do not allow sophisticated schedulability analysis techniques. In this paper we show how schedulability analysis can be integrated with object-oriented design. More specifically, we develop the schedulability and feasibility analysis method for the external messages that may suffer release jitter due to being dispatched by a tick driven scheduler in real-time control system, and we also develop the scheduliability method for sporadic activities, where message arrive sporadically then execute periodically for some bounded time. This method can be used to cope with timing constraints in realistic and complex real-time control systems. Using this method, a designer can quickly evaluate the impact of various implementation decisions on schedulability. In conjunction with automatic code-generation, we believe that this will greatly streamline the design and development of real-time control system software.
\end{abstract}

\section{Introduction}

There have been many attempts to make use of object-oriented technology for real-time software. Some of them have come from the industry real arena $[3,4,5]$, while others have come from academia $[6,7,8,9,10]$. Many of these claims are mostly based on assumption that real-time scheduling theory can be used to perform schedulability analysis. But, traditional real-time 
$42^{\text {nd }}$ IEEE Conference on Decision and Control, Maui, Hawaii, pp. 5932-5938, December 2003

scheduling theory results $[11,12,13,14]$ can be directly used only when the object models are restricted to look like the tasking models employed in real-time scheduling theory, as has been done in $[7,8]$. In other cases, either the claims are unsupported [4] or based on less sophisticated analysis $[4,6]$. Saksena and Karvels [15] provided the first attempt to apply real-time scheduling theory to the object-oriented design by use of the state-of the art in the both fields. In their paper, they show how to integrate traditional scheduliability analysis techniques with object-oriented design models based on the assumptions that the entire external message arrives perfectly on periodic or aperiodic time interval. Martins [17] provided the first attempts to commercially implement scheduling theory for UML model design by using the technologies in [15], these integrated tools allow issues on timeliness to be addressed much earlier on in the development process.

However, some critical issues regarding real-time control systems are not well addressed by the current approaches, especially because schedulability analysis for real-time control systems has not been effectively incorporated. Although some researchers $[15,16,17]$ have addressed this problems by providing code synthesis of scheduling aspects and functionality aspects models, they have mainly focused on the assumptions that all external events arrives perfectly on periodic or aperiodic without release jitter and sporadic effects. In general the real-time control systems are not the case, a message may be delayed by the polling of a tick scheduler, or perhaps awaiting the arrival of a message, and some real-time control systems have messages that behave as socalled sporadically periodic; a message arrival at some time, executes periodically for a bounded number of periods, and then re-arrives periodically for a number of times, and then does not rearrive for a larger time. Examples of such messages are interrupt handlers for burst interrupts or certain monitoring messages in real-time control systems. Until now there is no extended method of the object-oriented design methodologies to deal with these timing constraints of real-time control systems. Thus the above analysis methods need to be improved. 
$42^{\text {nd }}$ IEEE Conference on Decision and Control, Maui, Hawaii, pp. 5932-5938, December 2003

In this paper, we will present an approach to incorporating schedulability analysis in a UML for Real-Time (UML-RT) model-based development process [18]. Using this approach, satisfaction of the end-to-end timing constraints of real-time control systems can be verified and the schedulability analysis results will be used for aspect-oriented code generation in the model transformation and automatic code generation. The rest of the paper is organized as follows. In section 2, we briefly review basic concepts of UML-RT. Section 3 introduces schedulability analysis based on RMA. Section 4 develops the feasibility and schedulability analysis methods for real-time control systems with jitter messages and sporadically periodic messages. In section 5, we will present schedulability results for an example system based on our method. Finally we present some concluding remarks.

\section{Unified Modeling Language for Real-Time Systems}

The unified modeling language (UML) $[1,2]$ is a graphic modeling language for visualizing, specifying, constructing and documenting the artifacts of software systems. UML is a widely accepted language and it is becoming a standard for object-oriented modeling. UML has a strong set of general purpose modeling language concepts, and has been designed as an open-ended language application across different domains. UML-RT, developed by ObjectTime and Rational Rose Corporation, use UML to express the original ROOM (Real-Time Object-Oriented Modeling) concepts and their extensions.

\subsection{Structure Modeling}

UML-RT uses the notion of capsules to describe concurrent, active objects. Capsules are objects that communication with other capsules through interface called ports, and have each their own thread of execution. Capsules differ from other classes in that it can call operations on classes. Sending messages through public port is the only method that capsules can communicate with other capsules. Figure 1 shows an example of a systems structure for Automatic Gauge Control 
Systems in the tandem cold steel mill [19], consisting of several active objects, and interconnections between objects through ports.

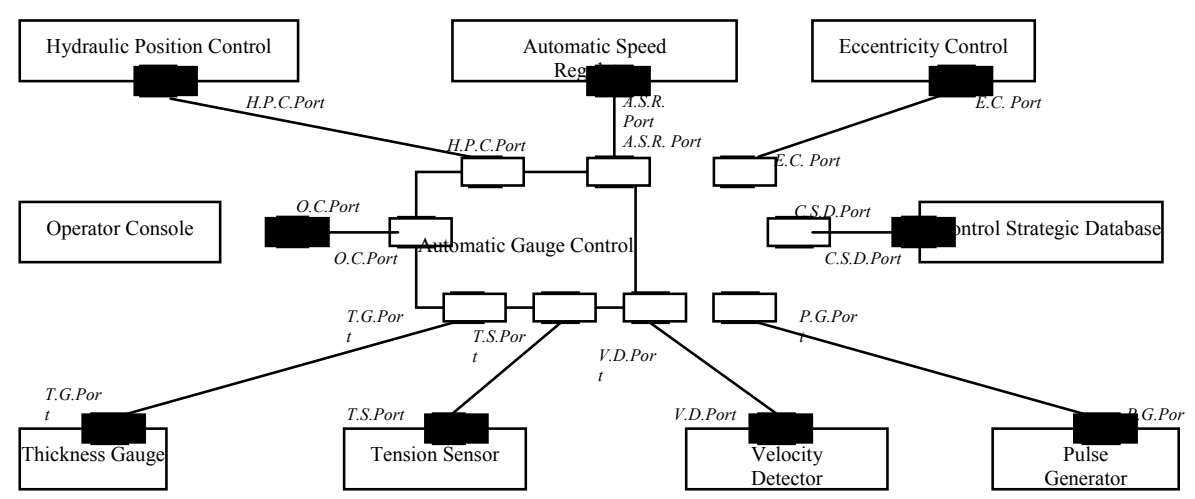

Figure 1. Object Structure Diagram for Automatic Gauge Control Systems

\subsection{Behavior Modeling}

In addition to the structure modeling, the capsules have their behavior defined by UML's hierarchical state machines and sequence diagrams. Sequence diagrams illustrate capsule interactions through message exchanges in a time sequence. Every capsule in the sequence diagram has a lifeline. Time progresses from top to bottom along a lifeline. The sequence diagrams use directed message arrows to describe messages sent from one capsule to another. The horizontal dimension represents the different objects in the interaction.

\section{Real-time Scheduling theory}

Scheduling theory for real-time systems has received a great deal of attention. The first contribution to real-time scheduling theory was made by Liu and Layland [11], they developed optimal static and dynamic priority scheduling algorithm for hard real-time sets of independent tasks. Since then, significant progresses have been made on generalizing and improving the schedulability analysis. The authors developed exact schedulability analysis to determine worstcase timing behavior for task with hard real-time constraints in the RMA model considered in the initial work [11], as well as extended models, such as arbitrary deadlines, release jitter, sporadic and periodic tasks $[12,13,14,20,21,22,23]$. 
$42^{\text {nd }}$ IEEE Conference on Decision and Control, Maui, Hawaii, pp. 5932-5938, December 2003

Most of the deterministic schedulability analysis techniques follow the same approach. First, the notion of the critical instant of a task is defined to be an instant at which a request for that task will have the largest response time. Then, the notion of busy period at level ' $i$ ' is defined to be a continuous interval of time during which events of priority ' $i$ ' or higher are being processed [11]. With these concepts, the calculation of the worst-case response time of an action involves the computation of the response time for successive arrivals of the action, starting from a critical instant until the end of the busy period, also the response time of a particular instant of action can be calculated by considering the effects of the blocking factor from lower priority actions and the interference factor from higher or equal priority actions, including the previous instance of the same action. If the worst-case response time of the action is less than or equal to it's deadline, the action can be said to be schedulable and feasible. Otherwise, the action is not schedulable or feasible.

\section{Schedulability Analysis and Extended Sequence Diagram of UML-RT}

\subsection{Analysis Model}

In our paper, we assume that real-time control systems are implemented in a uni-processor single thread environment, and it is made up of a set of transactions, where transaction denotes a single end-to-end computation within the system. Specifically, it refers to the entire causal set of actions executed as a result of the arrival of an external event that originated from an external source. External event sources are typically input devices (such as sensors) that interrupt the CPUrunning embedded software. These external events can be periodic or aperiodic, and also have jitter and sporadically periodic characteristics. We express the real-time control system as a collection of transactions that capture all computation in the design model. We also use the term action to capture the processing information associated with an external or internal event. In our model, an action captures this entire run-to-completion processing of an event. The execution of an action may generate internal events that trigger the execution of other actions. Thus, each 
transaction can be expressed as a collection of actions and events. Each action is a composite action, and composed from primitive sub-actions, these primitive sub-actions include send, call, and return actions [15], which generate internal events through sending messages to other objects. We use an extended sequence diagram from UML to describe transactions in the system models. In the extended sequence diagram, we capture the detail of the processing associated with an event. Figure 2 describes the transaction of automatic gauge control system in a steel mill. The transaction is driven by a timeout message with jitter characteristics. As can be seen, the automatic gauge control object obtains the steel plate thickness from the Thickness Gauge object using a synchronous call action. It then does the control law calculations and generates a position value, which is sent asynchronously to the hydraulic position control object, the hydraulic position control object then sends a command to the hydraulic position actuators adjusting the thickness of the steel plate. The sequence diagram for a transaction can easily be extended to include sub-actions associated with code executed by the real-time execution framework.

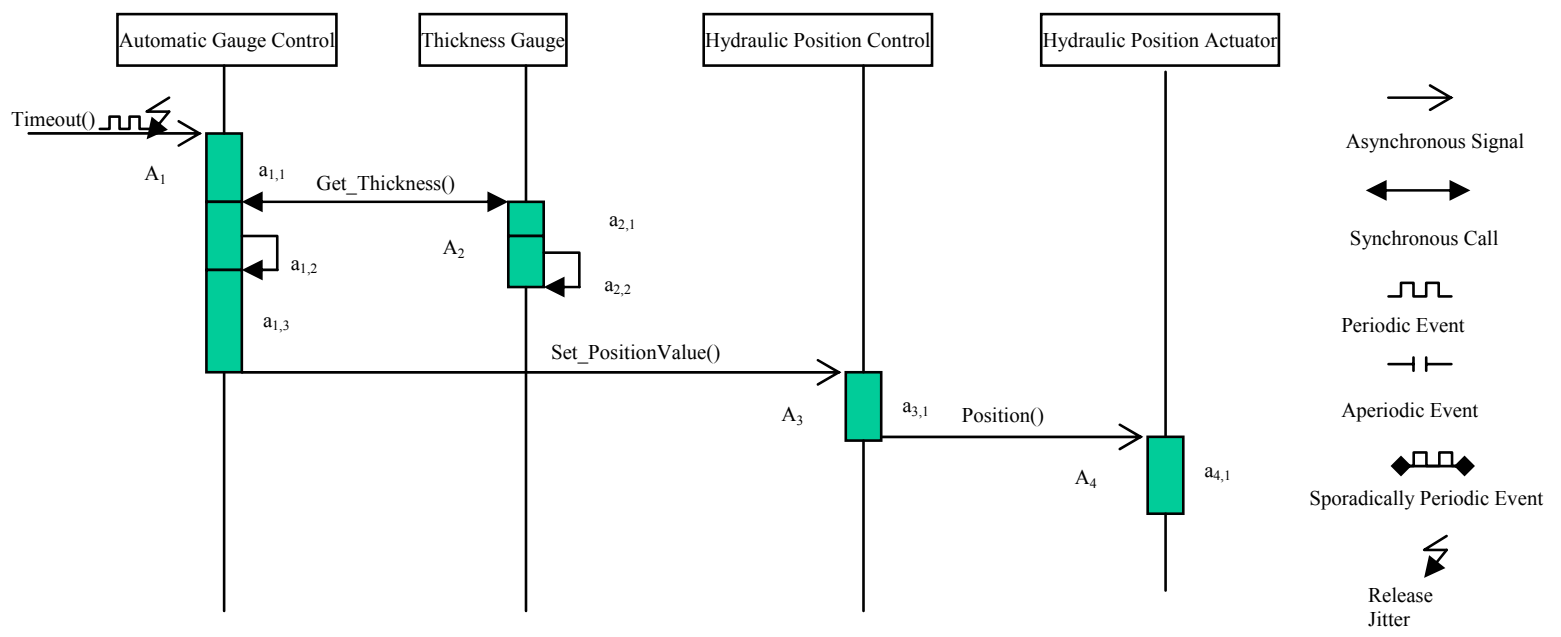

Figure 2. Extended Sequence Diagram of Automatic Gauge Control System

The extended sequence diagram can capture the timing constraints $[1,2]$. For the purpose of this paper, we are concerned about (1) arrival patterns of the external events, and (2) end-to-end deadlines of actions in the extended sequence diagram. The end-to-end deadlines can be specified on any action in a transaction, which is relative to the arrival of the external event. 
$42^{\text {nd }}$ IEEE Conference on Decision and Control, Maui, Hawaii, pp. 5932-5938, December 2003

\subsection{Notation}

In our paper, as defined in [15], we use event and message as synonymous. Let $\varepsilon=\left\{\mathrm{E}_{1}, \mathrm{E}_{2}, \mathrm{E}_{n}\right.$, $\left.\mathrm{E}_{n+1}, \ldots, \mathrm{E}_{N}\right\}$ represent the set of all event-streams in the system, where $\mathrm{E}_{1}, \mathrm{E}_{2}, \ldots, \mathrm{E}_{n}$ denote external event streams, and the remaining internal ones. All external events are assumed to be asynchronous, periodic, aperiodic events and sporadic events with release jitter. We use $\mathrm{J}_{i}$ to represent the jitter time of external event $\mathrm{E}_{i} \cdot \mathrm{T}_{i}$ and $\mathrm{t}_{i}$ represents the outer period and inner period for sporadically periodic external events $\mathrm{E}_{i}$. If the external event without sporadic effects, the inner period of such event is equal to it's outer period. Each external event stream $\mathrm{E}_{i}$ corresponds to a transaction $\tau_{i}$. We also use $\mathrm{A}_{i}$ to represent an action that associated with each event $\mathrm{E}_{i}$. An action may be decomposed into a sequence of sub-actions $\mathrm{A}_{i}=\left\{\mathrm{a}_{i, 1}, \mathrm{a}_{i, 2}, \mathrm{a}_{i, 3}, \ldots\right.$, $\mathrm{a}_{i, n_{i}}$, where each $\mathrm{a}_{i j}$ denotes a primitive action, such as sending message, calling message, and returning message. Within this model, each action $\mathrm{A}_{i}$ represents the entire "run-to-completion" processing associated with an event $\mathrm{E}_{i}$, and it is characterized as either asynchronously triggered or synchronously triggered, depending on whether the triggering event is asynchronous or synchronous. Each action $\mathrm{A}_{i}$ executes within the context of an active object (capsule) $\tilde{\mathrm{O}}\left(\mathrm{A}_{i}\right)$, and it is also characterized by a priority $\left(\pi\left(\mathrm{A}_{i}\right)\right)$, which is the same as the priority of its triggering event $\mathrm{E}_{\mathrm{i}}$. Each action $\mathrm{A}_{i}$ is also characterized by the computation time $\left(\mathrm{C}\left(\mathrm{A}_{i}\right)\right)$ and deadline ( $\mathrm{D}$ $\left(\mathrm{A}_{i}\right)$ ). Each sub-action $\mathrm{a}_{i j}$ of $\mathrm{A}_{i}$ is characterized by a computation time $\mathrm{C}\left(\mathrm{a}_{i j}\right)$ (abbreviated as $\mathrm{C}_{i j}$ ); the computation time of an action is simply the sum of its component sub-actions, i.e., $C\left(A_{i}\right)=\sum_{j} C_{i j}$, also, the computation time of any sequential sub-group of sub-actions $\mathrm{a}_{i p}$ to $\mathrm{a}_{i q}$ where $p \leq q$ is $C_{i, p \ldots q}=\sum_{j=p}^{j \leq q} C_{i j}$. Each event and action is part of a transaction. For the rest of this 
$42^{\text {nd }}$ IEEE Conference on Decision and Control, Maui, Hawaii, pp. 5932-5938, December 2003

paper, we use superscript to denote transactions. For example, $A_{i}^{\tau}$ represents an action and $E_{i}^{\tau}$ represents an event, both of which belong to transaction $\tau$. Adding the superscript for external events $\left\{\mathrm{E}_{k}: \mathrm{k}=1,2, \ldots, \mathrm{n}\right\}$ is unnecessary since there is exactly one external event associated with each transaction, i.e., external event $\mathrm{E}_{k}$ belongs to transaction $\mathrm{k}$ and would be denoted as $E_{k}^{k}$. In this case, the superscript will be omitted.

\section{Communication Relationships}

We assumed that there are two types of communication relationships between actions, asynchronous and synchronous. We use symbol " $\rightarrow$ " to denote asynchronous relationship. An asynchronous relationship $\mathrm{A}_{i} \rightarrow \mathrm{A}_{j}$ indicates that action $\mathrm{A}_{i}$ generates an asynchronous signal event $\mathrm{E}_{\mathrm{j}}$ (using a send sub-action) that triggers the execution of action $\mathrm{A}_{j}$. Likewise, we use symbol “↔” to denote synchronous relationship. A synchronous relationship $\mathrm{A}_{i} \leftrightarrow \mathrm{A}_{k}$ indicates that action $\mathrm{A}_{\mathrm{i}}$ generates a synchronous call event $\mathrm{E}_{k}$ (using a call sub-action) that triggers the execution of action $\mathrm{A}_{k}$. We assume that if the events have a synchronous relationship, the actions have the same priority. We also use a "causes" relationship, and use the symbol $\propto$ for that purpose. The relationship captures the causal relationship between actions. Both asynchronous and synchronous relationships are also causes relationships, i.e., $\mathrm{A}_{i} \rightarrow \mathrm{A}_{j}=>\left(\mathrm{A}_{i} \propto \mathrm{A}_{j}\right)$, and $\mathrm{A}_{i} \Leftrightarrow \mathrm{A}_{j}=>\left(\mathrm{A}_{i} \propto \mathrm{A}_{j}\right)$, Moreover, the causes relationship is transitive, thus $\left(\mathrm{A}_{i} \propto \mathrm{A}_{j}\right) \wedge$ $\left(\mathrm{A}_{j} \propto \mathrm{A}_{k}\right)=>\mathrm{A}_{i} \propto \mathrm{A}_{k}$. When $\mathrm{A}_{i} \propto \mathrm{A}_{j}$. We say that $\mathrm{A}_{j}$ is a successor of $\mathrm{A}_{i}$ since $\mathrm{A}_{i}$ must execute (at least partially) for $\mathrm{A}_{j}$ to be triggered.

\section{Synchronous Set}

For the purpose of analysis, we define the term "synchronous set of $\mathrm{A}_{i}$ ". The synchronous set of $\mathrm{A}_{i}$ is a set of actions that can be built starting from action $\mathrm{A}_{i}$ and adding all actions that are 
$42^{\text {nd }}$ IEEE Conference on Decision and Control, Maui, Hawaii, pp. 5932-5938, December 2003

called synchronously from it. The process is repeated recursively until no more actions can be added to the list. In there, we use $\Upsilon\left(\mathrm{A}_{i}\right)$ denote the synchronous set of $\mathrm{A}_{\mathrm{i}}$ and $\mathrm{C}\left(\Upsilon\left(\mathrm{A}_{i}\right)\right)$ denote the cumulative execution time of all the actions in this synchronous set. We also call $\mathrm{A}_{i}$ as the root action of this synchronous set.

\subsection{A Simple Example}

We will use a simple example system shown in Table 1 through the rest of this paper to illustrate our ideas. The extended system sequence diagram is shown in Figure 3. The example system consists of three transactions triggered by external events $\mathrm{E}_{i}$, one is periodic event with release jitter, one is sporadically periodic event, and the other one is aperiodic with release jitter. All the transactions are statically assigned to a single thread. For each action, we show the sub-actions $\mathrm{a}_{i j}$, their computation times as well as which internal events are generated by which sub-action.

Note that within each transaction we have included both synchronous (call) and asynchronous (signal) events. Furthermore, each transaction traverses multiple objects, and has multiple priorities (due to different deadlines for different parts of the transaction).

\begin{tabular}{|c|c|c|c|c|c|c|c|c|c|c|c|c|}
\hline $\begin{array}{c}\text { Trans } \\
\tau_{\mathrm{i}}\end{array}$ & $\begin{array}{c}\text { Out.P. } \\
T_{i}\end{array}$ & $\begin{array}{c}\text { Inn.P. } \\
t_{i}\end{array}$ & $\begin{array}{c}\text { Num. } \\
n_{i}\end{array}$ & $\begin{array}{c}\text { Jitter } \\
\mathrm{J}_{\mathrm{i}}\end{array}$ & $\begin{array}{c}\text { Event(Type) } \\
E_{i}\end{array}$ & $\begin{array}{c}\text { Action } \\
\mathrm{A}_{\mathrm{i}}\end{array}$ & $\begin{array}{c}\text { Priority } \\
\pi\left(\mathrm{A}_{\mathrm{i}}\right)\end{array}$ & $\begin{array}{c}\text { Deadline } \\
\mathrm{D}\left(\mathrm{A}_{\mathrm{i}}\right)\end{array}$ & $\begin{array}{l}\text { Object } \\
\tilde{O}\left(A_{i}\right)\end{array}$ & $\begin{array}{l}\text { Sub-action } \\
\mathrm{a}_{\mathrm{ij}}\end{array}$ & $\begin{array}{c}\text { Comp.Time } \\
\mathrm{c}_{\mathrm{ij}}\end{array}$ & $\begin{array}{l}\text { Events Generated } \\
\qquad E_{i}\left(a_{i, j}\right)\end{array}$ \\
\hline$\tau_{1}$ & 60 & 60 & 1 & 10 & $\begin{array}{l}\mathrm{E}_{1} \text { (External) } \\
\mathrm{E}_{4} \text { (Signal) } \\
\mathrm{E}_{5}(\text { Signal }) \\
\mathrm{E}_{6}(\text { Call })\end{array}$ & $\begin{array}{l}\mathrm{A}_{1} \\
\mathrm{~A}_{4} \\
\mathrm{~A}_{5} \\
\mathrm{~A}_{6}\end{array}$ & $\begin{array}{c}10 \\
6 \\
10 \\
10\end{array}$ & $\begin{array}{l}300 \\
800 \\
300 \\
280\end{array}$ & $\begin{array}{l}1 \\
4 \\
3 \\
4\end{array}$ & $\begin{array}{l}\left\{a_{1,1}, a_{1,2}, a_{1,3}\right\} \\
\left\{a_{4,1}\right\} \\
\left\{a_{5,1}, a_{5,2}, a_{5,3,} a_{5,4}\right\} \\
\left\{a_{6,1}, a_{6,2}\right\}\end{array}$ & $\begin{array}{l}\{5,1,1\} \\
\{5\} \\
\{2,1,2,1\} \\
\{4,1\}\end{array}$ & $\begin{array}{l}\mathrm{E}_{4}\left(\mathrm{a}_{1,2}\right), \mathrm{E}_{5}\left(\mathrm{a}_{1,3}\right) \\
\ldots \\
\mathrm{E}_{6}\left(\mathrm{a}_{5,2}\right) \\
\ldots\end{array}$ \\
\hline$\tau_{2}$ & 900 & 300 & 3 & & $\begin{array}{l}\mathrm{E}_{2} \text { (External) } \\
\mathrm{E}_{7} \text { (Call) } \\
\mathrm{E}_{8}(\text { Signal }) \\
\mathrm{E}_{9}(\text { Call })\end{array}$ & $\begin{array}{l}\mathrm{A}_{2} \\
\mathrm{~A}_{7} \\
\mathrm{~A}_{8} \\
\mathrm{~A}_{9}\end{array}$ & $\begin{array}{l}9 \\
9 \\
7 \\
9\end{array}$ & $\begin{array}{l}460 \\
400 \\
720 \\
450\end{array}$ & $\begin{array}{l}2 \\
5 \\
4 \\
6\end{array}$ & $\begin{array}{l}\left\{\mathrm{a}_{2,1}, \mathrm{a}_{2,2}, \mathrm{a}_{2,3}, \mathrm{a}_{2,4}, \mathrm{a}_{2,5}\right\} \\
\left\{\mathrm{a}_{7,1}, \mathrm{a}_{7,2}\right\} \\
\left\{\mathrm{a}_{8,1}\right\} \\
\left\{\mathrm{a}_{9,1} \mathrm{a}_{9,2}\right\}\end{array}$ & $\begin{array}{l}\{1,3,1,1,4\} \\
\{9,1\} \\
\{10\} \\
\{50,1\}\end{array}$ & $\begin{array}{l}E_{7}\left(a_{2,1}\right), E_{8}\left(a_{2,3}\right) E_{9}\left(a_{2,4}\right) \\
-- \\
\cdots \\
--\end{array}$ \\
\hline$\tau_{3}$ & 1000 & 1000 & 1 & 5 & $\begin{array}{l}\mathrm{E}_{3} \text { (External) } \\
\mathrm{E}_{10}(\text { Call }) \\
\mathrm{E}_{11}(\text { Signal })\end{array}$ & $\begin{array}{l}\mathrm{A}_{3} \\
\mathrm{~A}_{10} \\
\mathrm{~A}_{11}\end{array}$ & $\begin{array}{l}8 \\
8 \\
5\end{array}$ & $\begin{array}{l}620 \\
600 \\
480\end{array}$ & $\begin{array}{l}3 \\
6 \\
7\end{array}$ & $\begin{array}{l}\left\{a_{3,1}, a_{3,2}, a_{3,3}\right\} \\
\left\{a_{10,1}, a_{10,2}, a_{10,3,} a_{10,4}\right\} \\
\left\{a_{11,1}\right\}\end{array}$ & $\begin{array}{l}\{4,1,5\} \\
\{4,1,5,1\} \\
\{250\}\end{array}$ & $\begin{array}{l}E_{10}\left(a_{3,2}\right) \\
E_{11}\left(a_{10,2}\right) \\
---\end{array}$ \\
\hline
\end{tabular}

Table 1. An Example System For Schedulability And Feasibility Analysis

In our example system, events have unique priorities (termed the priority); events can arrive at any time (i.e. want to run), but can be delayed for a variable bounded amount of time (termed the release jitter) before being placed in a priority-ordered run-queue. Periodic and aperiodic events 
are given worst-case inter-arrival time (termed the period); and sporadically periodic events are given the outer period and inner period, a event cannot re-arrive sooner than this time, for each arrival a event may execute a bounded amount of computation, each event is associated with the action, each action is given the worst-case execution time and deadline, This worst-case execution time value is deemed to contain the overhead due to context switching. The cost of preemption, within the model, is thus assumed to be zero.

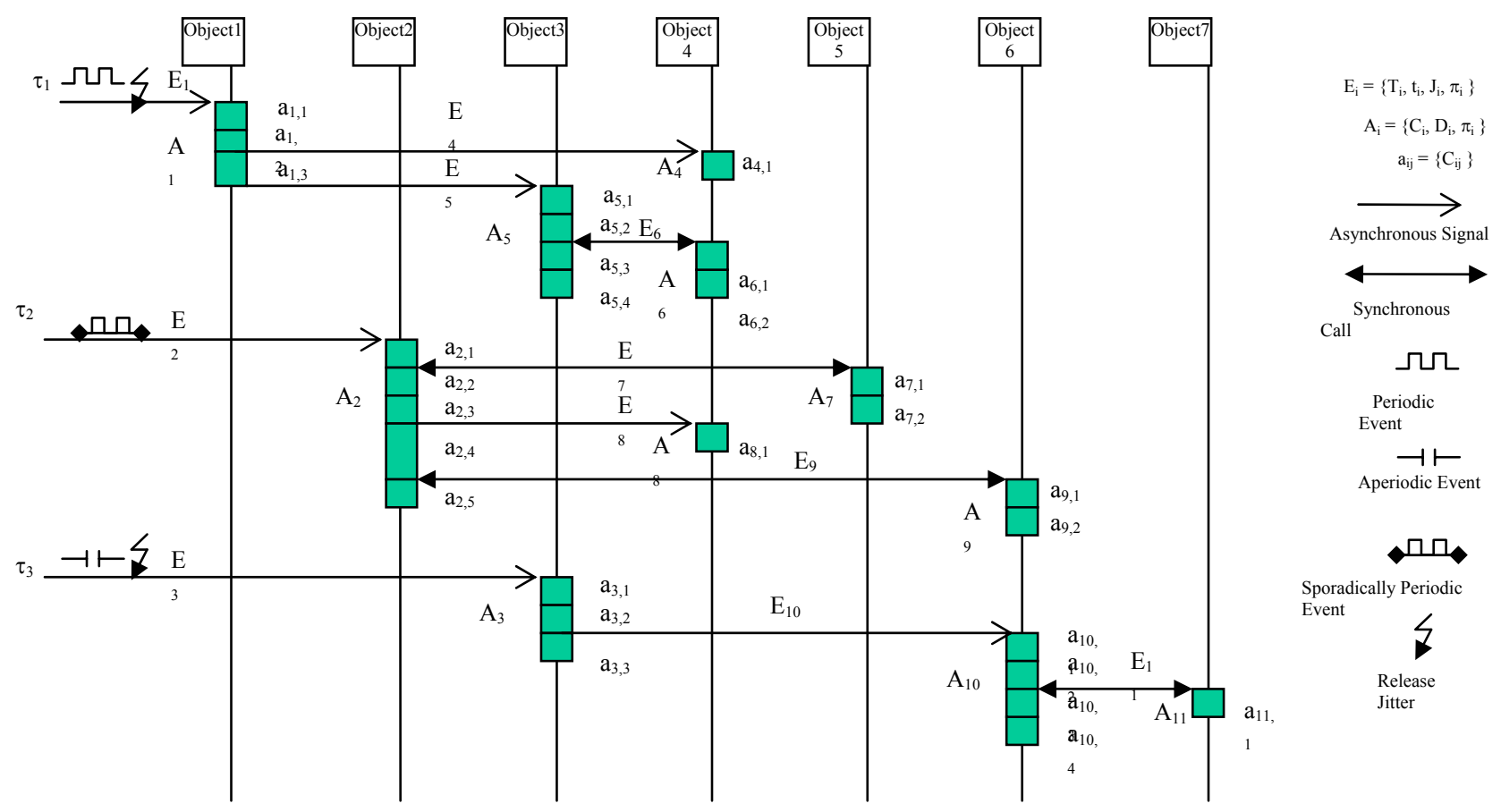

Figure 3. Extended Sequence Diagram of The Example System

\section{4. Schedulability and Feasibility Analysis}

In our real-time control system model, we assume that only the external event has release jitter problem, and the internal event does not have jitter problem, because the internal event arrival is only decided by the action that represents the entire "run-to-completion" processing associated with the internal event. For the external events $\mathrm{E}_{\tau}$ which behave as 'sporadically periodic' executing with an inner period $\left(\mathrm{t}_{\tau}\right)$ and outer period $\left(\mathrm{T}_{\tau}\right)$. we assume that the 'burst' behavior must finish before the next burst (i.e., $n_{\tau} t_{\tau} \leq T_{\tau}$ ), where $\mathrm{n}_{\tau}$ is the number of release of external 
$42^{\text {nd }}$ IEEE Conference on Decision and Control, Maui, Hawaii, pp. 5932-5938, December 2003

events $\mathrm{E}_{\tau}$ in a burst, and also we assumed that the release jitter $\left(\mathrm{J}_{\tau}\right)$ of external event $\mathrm{E}_{\tau}$ is the inner release jitter (i.e., each release of external events $\mathrm{E}_{\tau}$ can suffer this jitter). In our analysis model, we carry out the schedulability and feasibility analysis by calculating the worst-case response time of actions, the worst-case response time of actions $\mathrm{A}_{i}^{\tau}$ is calculated relative to the arrival of the external event $\mathrm{E}_{\tau}$ that triggers the transaction $\tau$. If the worst-case response time of an action is less than or equal to it's deadline, the action is schedulable, if all the worst-case times of actions in the systems are less than or equal to their deadline; the system is schedulable or feasible. We use the well-known critical instant/busy-period analysis $[6,11,12,14]$ developed for fixed priority scheduling, In our uni-processor single thread implementation environments, a priority inversion occurs if a lower priority event is processed, while a higher priority event is pending. In the same way, a level-i busy period is a continuous interval of time during which events of priority " $i$ ” or higher are being processed.

\subsubsection{Worst-Case Response Time Analysis}

In the worst-case response time analysis for action $\mathrm{A}_{i}^{\tau}$, we will compute the response time of the action for successive arrivals of the transaction, staring from a critical instant, until the end of the busy period. We let $S_{i}^{\tau}(q)$ denote the worst-case start time for instance ' $q$ ' of action $\mathrm{A}_{i}^{\tau}$ (i.e., when the instance ' $q$ ' of the action gets the CPU for the first time), starting from the critical instant (time 0). Likewise, $F_{i}^{\tau}(q)$ denotes the worst-case finish time, starting from the critical instant (time 0). $\mathrm{A}_{\mathrm{rr \tau}}(q)$ denotes the arrival time of instance ' $q$ ' of external event $\mathrm{E}_{\tau}$ starting from the critical instant (time 0 ). According to our system model, we not only consider the busy-period starting at time $\mathrm{J}_{\tau}+q \mathrm{~T}_{\tau}$, but also consider busy-period starting at $\mathrm{J}_{\tau}+q \mathrm{t}_{\tau}$ before the release of event $\mathrm{E}_{\tau}$. In order to do that, we define two integers $\mathrm{M}_{\tau}$ and $\mathrm{m}_{\tau}$, where $\mathrm{M}_{\tau}$ is the number of 
$42^{\text {nd }}$ IEEE Conference on Decision and Control, Maui, Hawaii, pp. 5932-5938, December 2003

outer periods previously in the window $\left[0, S_{i}^{\tau}(q)\right]$, and $\mathrm{m}_{\tau}$ is the number of inner periods. $\mathrm{M}_{\tau}$ and $\mathrm{m}_{\tau}$ are given by:

$$
\begin{aligned}
& \mathrm{M}_{\tau}=\left\lfloor\frac{q-1}{n_{\tau}}\right\rfloor \\
& \mathrm{m}_{\tau}=(\mathrm{q}-1)-\mathrm{M}_{\tau} \mathrm{m}_{\tau}
\end{aligned}
$$

Where $q$ is an integer, and $q \geq 1$.

The arrival time $\mathrm{A}_{\mathrm{rr \tau}}(q)$ of instance ' $q$ ' of external event $\mathrm{E}_{\tau}$ can be given as $\mathrm{A}_{\mathrm{rr \tau}}(q)=$ $\mathrm{M}_{\tau} \mathrm{T}_{\tau}+\mathrm{m}_{\tau} \mathrm{t}_{\tau}$. Base on the traditional scheduling theory for real time systems $[11,12,13,14]$, we can iteratively compute $S_{i}^{\tau}(q)$ and $F_{i}^{\tau}(q)$ for $q=1,2,3 \ldots$ until we reach a $q=m$, such that $F_{i}^{\tau}(q) \leq \mathrm{A}_{\mathrm{rr} \tau}(m+1)-\mathrm{J}_{\tau}$. Then, we let $\mathrm{R}\left(\mathrm{A}_{i}^{\tau}\right)$ denote the worst-case response time of action $\mathrm{A}_{i}^{\tau}$, and it is given by:

$$
\mathrm{R}\left(\mathrm{A}_{i}^{\tau}\right)=\max _{q \in[1,2, \ldots, m]}\left\{F_{i}^{\tau}(q)+\mathrm{J}_{\tau}-\operatorname{Arr}_{\tau}(q)\right\}
$$

\subsubsection{Blocking}

According the scheduling theory $[11,15]$, blocking refers to the effect of lower priority actions on the response time of an action. It may be from any transaction. Let $\mathrm{B}\left(\mathrm{A}_{i}^{\tau}\right)$ denote the maximum blocking time of an action $\mathrm{A}_{i}^{\tau}$, In uni-processor single-thread implementation environments, since scheduling is non-preemptive, priority inversion is limited to one synchronous set of actions with a lower priority root action. This action has started executing just before the transaction containing $\mathrm{A}_{i}^{\tau}$ arrives. Thus the maximum blocking time of an action is given by:

$$
\mathbf{B}\left(\mathbf{A}_{i}^{\tau}\right)=\max _{1 \leq k \leq N}\left\{\mathbf{C}\left(\Upsilon\left(\mathbf{A}_{k}\right)\right):: \pi\left(\mathbf{A}_{i}^{\tau}\right) \geq \pi\left(\mathbf{A}_{k}\right)\right\}
$$

\subsubsection{Interference Effects and Busy Period Analysis}


$42^{\text {nd }}$ IEEE Conference on Decision and Control, Maui, Hawaii, pp. 5932-5938, December 2003

We known that the critical instant of an action $\mathrm{A}_{i}^{\tau}$ occurs when all transaction arrive at the same time (we denote this as time 0), and the root action of the synchronous set of actions that contributes the maximum blocking term $\mathrm{B}\left(\mathrm{A}_{i}^{\tau}\right)$. Since actions are executed in a non-preemptive manner, when $\mathrm{A}_{i}^{\tau}$ starts executing, no other action can interrupt it other than any synchronous calls that $\mathrm{A}_{i}^{\tau}$ makes. Firstly, let the early interference function $\operatorname{Early}_{k}^{A^{\tau}(q)}(\mathrm{t})$ denote the interference effect of transaction $k$ prior to $S_{i}^{\tau}(q)$, assuming that $S_{i}^{\tau}(q)=$ t. Likewise, let the late interference function Late ${ }_{k}^{A^{\tau}(q)}(\mathrm{t})$ denote the interference effect of transaction $k$ for the interval $\left[S_{i}^{\tau}(q), F_{i}^{\tau}(q)\right)$, assuming that $F_{i}^{\tau}(q)=$ t. Then, the value for $S_{i}^{\tau}(q)$ is given by the lowest value of $\mathrm{W}_{i}^{\tau}(q)$, it satisfies the following equation.

$$
S_{i}^{\tau}(q)=\min \mathbf{W}_{i}^{\tau}(q):: \mathbf{W}_{i}^{\tau}(q)=\mathbf{B}\left(\mathbf{A}_{i}^{\tau}\right)+\sum_{1 \leq k \leq N} \operatorname{Early}_{k}^{A^{\tau}(q)}\left(\mathbf{W}_{i}^{\tau}(q)\right)
$$

That is, an action (instance) will start, in the worst case, at a time $\mathrm{W}_{i}^{\tau}(q)$ if the sum of the blocking and interference effects equals $\mathrm{W}_{i}^{\tau}(q)$, where $\mathrm{W}_{i}^{\tau}(q)$ is the first time instant when this become true. Note that the term $\mathrm{W}_{i}^{\tau}(q)$ occurs on both sides of the equation, this equation can be solved by iteratively refining $\mathrm{W}_{i}^{\tau}(q)$ using the right side of the equation, starting from an initial lower bound value $\mathrm{B}\left(\mathrm{A}_{i}^{\tau}\right)$ in this case, as explained in $[11,15,21]$.

Once $S_{i}^{\tau}(q)$ is known, we can compute $F_{i}^{\tau}(q)$, this is done by considering the additional interference effects from higher or equal priority actions that can preempt $\mathrm{A}_{i}^{\tau}(q)$. Because in our uni-processor single thread implementation system model, there can be no preemption effects after an action has started executing, thus we have $\operatorname{Late}_{k}^{A^{\tau}(q)}(\mathrm{t})=0$. So, $F_{i}^{\tau}(q)$ can be calculated as follow: 
$42^{\text {nd }}$ IEEE Conference on Decision and Control, Maui, Hawaii, pp. 5932-5938, December 2003

$$
F_{i}^{\tau}(q)=S_{i}^{\tau}(q)+\mathbf{C}\left(\Upsilon\left(\mathbf{A}_{i}^{\tau}\right)\right)
$$

Where $\mathrm{C}\left(\Upsilon\left(\mathrm{A}_{i}^{\tau}\right)\right)$ is the cumulative execution time of all the actions in this synchronous set of $\mathrm{A}_{i}^{\tau}$.

\subsubsection{Early Interference Function.}

The early interference function depends on whether we are considering interference from other different transaction $\mathrm{K} \neq \tau$, or from the same transaction. i.e., $\mathrm{K}=\tau$.

Early Interference effects from Other Different Transactions. In this case $K \neq \tau$, for any arrival of the transaction $k$ in the interval $\left[0, \mathrm{~W}_{i}^{\tau}(q)\right]$. We have to consider the computation times of all higher or equal priority actions making up transaction $k$, again, any synchronous call made recursively from these actions must also be considered, we can see that they have been already included in the calculation because of our earlier assumption that the priority of a synchronously triggered action is the same as that of the caller action. Also, interference is considered for all events arrived in the window $\left[0, \mathrm{~W}_{i}^{\tau}(q)\right]$. Note that we have to take the closed interval, because if a higher action becomes enabled at time $\mathrm{W}_{i}^{\tau}(q)$, then $\mathrm{A}_{i}^{\tau}(q)$ cannot begin executing. Now consider the computation occurring in the window $\left[0, \mathrm{~W}_{i}^{\tau}(q)\right]$ from higher priority sporadically periodic event $\mathrm{E}_{k}$ with release jitter $\mathrm{J}_{k}$, if the window is larger than a number of 'bursts' of $\mathrm{E}_{k}$ then the computation time from each burst amount is $\mathrm{n}_{k} \mathrm{C}\left(\mathrm{A}_{k}\right)$. For the partial 'burst' starting in the window, we can treat $\mathrm{E}_{k}$ as a simple periodic event executing with period $\mathrm{t}_{k}$ over the remaining part of the window. We let $\mathrm{F}_{K}$ represents the whole number of event $\mathrm{E}_{k}$ 'bursts' starting and finishing in the window, and it is given as follow: 
$42^{\text {nd }}$ IEEE Conference on Decision and Control, Maui, Hawaii, pp. 5932-5938, December 2003

$$
\mathbf{F}_{k}=\left\lfloor\frac{J_{k}+W_{i}^{\tau}(q)}{T_{k}}\right\rfloor
$$

The remaining part of the window $\left[0, \mathrm{~W}_{i}^{\tau}(q)\right]$ is the length $J_{K}+W_{i}^{\tau}(q)-F_{k} T_{k}$, hence a bound on the number of event $\mathrm{E}_{k}$ in this remaining time is $\mathrm{F}_{k r}$, and it is given by:

$$
\mathbf{F}_{k r}=\left\lfloor\frac{J_{K}+W_{I}^{\tau}(q)-F_{K} T_{K}}{t_{K}}\right\rfloor+1
$$

Another bound on the number of event $\mathrm{E}_{k}$ in this remaining time is $\mathrm{n}_{k}$, since a burst can consist of at most $\mathrm{n}_{k}$ invocations of event $\mathrm{E}_{k}$. Therefore the least upper bound number $\mathrm{F}_{k r \text { min }}$ can be given by:

$$
\mathbf{F}_{k r \min }=\min \left(\mathrm{n}_{k}, \mathbf{F}_{k r}\right)
$$

So the total interference of action $\mathrm{A}_{i}^{\tau}$ from different other transaction $k$ is given as:

$$
\operatorname{Early}_{k \neq \tau}^{A^{\tau}(q)}\left(\mathbf{W}_{i}^{\tau}(q)\right)=\left(\mathbf{F}_{k r \min }+\mathbf{F}_{K} \mathbf{n}_{k}\right) \bullet \sum_{l}\left(c\left(A_{l}^{k}\right):: \pi\left(A_{l}^{k}\right) \geq \pi\left(A_{i}^{\tau}\right)\right)
$$

Early Interference effects from The Same Transactions. In this case $\mathrm{K}=\tau$, it is important to distinguish between previous instance, i.e., $1,2, \ldots, q-1$ of the transaction, and all other instances after that. Accordingly, we can write;

$$
\operatorname{Early}_{\tau}^{A^{\tau}(q)}\left(\mathrm{W}_{i}^{\tau}(q)\right)=\operatorname{Early}_{\tau^{-}}^{A^{\tau}(q)}\left(\mathrm{W}_{i}^{\tau}(q)\right)+\operatorname{Early}_{\tau^{+}}^{A^{\tau}(q)}\left(\mathrm{W}_{i}^{\tau}(q)\right)
$$

Where the Early ${ }_{\tau^{-}}^{A^{\tau}(q)}\left(\mathrm{W}_{i}^{\tau}(q)\right)$ is the interference effects from the past instances $(1,2, \ldots, q-1)$ and Early ${ }_{\tau^{+}}^{A^{\tau}(q)}\left(\mathrm{W}_{i}^{\tau}(q)\right)$ is the interference effects of all other instances $q, q+1, \ldots$ that may have arrived in $\left[0, S_{i}^{\tau}(q)\right]$.

The past instances of the transaction have similar effects as other transactions, since any higher or equal priority actions of the transaction must execute prior to $\mathrm{A}_{i}^{\tau}(q)$. Thus the

Early $_{\tau^{-}}^{A^{\tau}(q)}\left(\mathrm{W}_{i}^{\tau}(q)\right.$ can be given as: 
$42^{\text {nd }}$ IEEE Conference on Decision and Control, Maui, Hawaii, pp. 5932-5938, December 2003

$$
\operatorname{Early}_{\tau^{-}}^{A^{\tau}(q)}\left(\mathbf{W}_{i}^{\tau}(q)\right)=\left(M_{\tau} n_{\tau}+m_{\tau}\right) \bullet \sum_{l}\left(C\left(A_{l}^{\tau}\right):: \pi\left(\mathbf{A}_{l}^{\tau}\right) \geq \pi\left(\mathbf{A}_{i}^{\tau}\right)\right)
$$

The interference effect of instance $q$ onwards must not count the effect of any action $\mathrm{A}_{l}^{\tau}$, if

$\mathrm{A}_{i}^{\tau} \propto \mathrm{A}_{l}^{\tau}$, since if $\mathrm{A}_{i}^{\tau}(q)$ has not executed, any action that is caused by it could not have executed either. Furthermore, we assume that multiple instances of the same action execute in order and thus, this is true for instance $q+1$ onward as well.

If the action $\mathbf{A}_{i}^{\tau}$ is asynchronously triggered, the $\operatorname{Early}_{\tau^{+}} A^{\tau}(q)\left(\mathrm{W}_{i}^{\tau}(q)\right)$ is given by the following equations:

First, let $\mathrm{F}_{\tau}$ represent the whole number of event $\mathrm{E}_{\tau}$ 'bursts' starting and finishing in the window $\left[0, \mathrm{~W}_{i}^{\tau}(q)\right]$ and is given by:

$$
\mathrm{F}_{\tau}=\left\lfloor\frac{W_{i}^{\tau}(q)}{T_{\tau}}\right\rfloor
$$

The remaining part of the window $\left[0, \mathrm{~W}_{i}^{\tau}(q)\right]$ is the length $W_{i}^{\tau}(q)-F_{\tau} T_{\tau}$, hence a bound on the number of event $\mathrm{E}_{\tau}$ in this remaining time is $\mathrm{F}_{\tau r}$, and it is given by:

$$
F_{\tau r}=\left\lfloor\frac{W_{i}^{\tau}(q)-F_{\tau} T_{\tau}}{t_{\tau}}\right\rfloor+1
$$

Another bound on the number of event $\mathrm{E}_{\tau}$ in this remaining time is $\mathrm{n}_{\tau}$, since a burst can consist of at most $n_{\tau}$ invocations of event $E_{\tau}$. Therefore the least upper bound number $\mathrm{F}_{\tau r \min }$ can be given by:

$$
\mathbf{F}_{\tau r \min }=\min \left(\mathrm{n}_{\tau}, \mathbf{F}_{\tau r}\right)
$$

So, the Early ${ }_{\tau^{+}}^{A^{\tau}(q)}\left(\mathrm{W}_{i}^{\tau}(q)\right)$ is given by: 


$$
\begin{aligned}
& \operatorname{Early}_{\tau^{+}}^{A^{\tau}(q)}\left(\mathbf{W}_{i}^{\tau}(q)\right)=\left\{\left(\mathbf{F}_{\tau r \text { min }}+\mathbf{F}_{\tau} \mathrm{n}_{\tau}\right)-\right. \\
&\left.\left(M_{\tau} n_{\tau}+m_{\tau}\right)\right\} \bullet\left(\sum _ { l } \left(C\left(A_{l}^{\tau}\right):: \neg\left(A_{i}^{\tau} \propto A_{l}^{\tau}\right) \wedge \pi\left(A_{l}^{\tau}\right) \geq \pi\left(A_{i}^{\tau}\right)\right.\right.
\end{aligned}
$$

According to the above analysis, for the asynchronously triggered action $\mathrm{A}_{i}^{\tau}$, we can find start times $S_{i}^{\tau}(q)$ as follows:

$$
\begin{aligned}
S_{i}^{\tau}(q)= & \min \mathbf{W}_{i}^{\tau}(q):: \\
\mathbf{W}_{i}^{\tau}(q)= & \mathbf{B}\left(\mathbf{A}_{i}^{\tau}\right)+\sum_{1 \leq k \leq N} \operatorname{Early}_{k}^{A_{i}^{\tau}(q)}\left(\mathbf{W}_{i}^{\tau}(q)\right) \\
= & \mathbf{B}\left(\mathbf{A}_{i}^{\tau}\right) \\
& +\sum_{k \neq \tau}\left(F_{k r \min }+F_{k} n_{k}\right) \sum_{l}\left(c\left(A_{l}^{K}\right):: \pi\left(\mathbf{A}_{l}^{k}\right) \geq \pi\left(\mathbf{A}_{i}^{\tau}\right)\right) \\
& +\left(M_{\tau} n_{\tau}+m_{\tau}\right) \bullet \sum_{l}\left(C\left(A_{l}^{\tau}\right):: \pi\left(\mathbf{A}_{l}^{\tau}\right) \geq \pi\left(\mathbf{A}_{i}^{\tau}\right)\right) \\
+ & \left\{\left(\mathbf{F}_{\tau r \min }+\mathbf{F}_{\tau} \mathrm{n}_{\tau}\right)-\left(M_{\tau} n_{\tau}+m_{\tau}\right)\right\} \bullet\left(\sum _ { l } \left(C\left(A_{l}^{\tau}\right):: \neg\left(A_{i}^{\tau} \propto A_{l}^{\tau}\right) \wedge \pi\left(A_{l}^{\tau}\right) \geq \pi\left(A_{i}^{\tau}\right)\right.\right.
\end{aligned}
$$

If the action $\mathbf{A}_{i}^{\tau}$ is synchronously triggered, the above worst staring time $S_{i}^{\tau}(q)$ for the asynchronously triggered action $\mathrm{A}_{i}^{\tau}$ need to be improved. Now, let's consider a synchronously triggered action $\mathrm{A}_{i}^{\tau}$, let $\mathrm{A}_{g}^{\tau}$ be the asynchronously triggered action, such that $\mathrm{A}_{i}^{\tau}$ belongs to $\Upsilon\left(A_{g}^{\tau}\right)$, i.e., the synchronous-set of $A_{g}^{\tau}$. Then we have a chain of actions, starting from $\mathrm{A}_{g}^{\tau}$ to $\mathrm{A}_{i}^{\tau}$ that only execute partially in this interval, and are blocked waiting for $\mathrm{A}_{i}^{\tau}$ to execute. Note that there must be exactly one such action $A_{g}^{\tau}$, so there is no ambiguity. This changes the interference for instances $q, q+1, \ldots$ of transaction $\tau$. For instance $q$, only a part of the synchronous set $\Upsilon\left(A_{g}^{\tau}\right)$ has executed, and 
$42^{\text {nd }}$ IEEE Conference on Decision and Control, Maui, Hawaii, pp. 5932-5938, December 2003

this should be reflected in the equation. Rather than extend the notation to explicitly define this subset. We denote this sub-action as $\mathrm{a}_{g, h}^{\tau}$ producing the action $\mathrm{A}_{i}^{\tau}$, and the computation time associated with this sub-action as $C\left(\operatorname{sub}\left(\gamma\left(a_{g, 1 \ldots h}^{\tau}\right)\right)\right)$. For instances $\mathrm{q}+1$ onwards, none of the actions in the synchronous set $\Upsilon\left(A_{g}^{\tau}\right)$ can cause interference, since their previous instance $(q)$ is blocked. The blocking term, interference from other transaction, and interference from previous instances $(0,1,2, \ldots, q-1)$ of the same transaction remain the same, because we assumed that $\pi\left(\mathrm{A}_{g}^{\tau}\right)=\pi\left(\mathrm{A}_{i}^{\tau}\right)$. Based the above analysis, the worst staring time $S_{i}^{\tau}(q)$ for the synchronously triggered action $\mathrm{A}_{i}^{\tau}$ is given as follows

$$
\begin{aligned}
& S_{i}^{\tau}(q)=\min \mathbf{W}_{i}^{\tau}(q):: \\
& \mathbf{W}_{i}^{\tau}(q)=\mathbf{B}\left(\mathbf{A}_{g}^{\tau}\right)+\sum_{1 \leq k \leq N} \operatorname{Early}_{k}^{A_{i}^{\tau}(q)}\left(\mathbf{W}_{i}^{\tau}(q)\right) \\
& =\mathbf{B}\left(\mathbf{A}_{g}^{\tau}\right) \\
& +\sum_{k \neq \tau}\left(F_{k r \min }+F_{k} n_{k}\right) \sum_{l}\left(c\left(A_{l}^{K}\right):: \pi\left(\mathbf{A}_{l}^{k}\right) \geq \pi\left(\mathbf{A}_{g}^{\tau}\right)\right) \\
& +\left(M_{\tau} n_{\tau}+m_{\tau}\right) \bullet \sum_{l}\left(C\left(A_{l}^{\tau}\right):: \pi\left(\mathbf{A}_{l}^{\tau}\right) \geq \pi\left(\mathbf{A}_{g}^{\tau}\right)\right) \\
& +C\left(\operatorname{sub}\left(\gamma\left(a_{g, 1 \ldots h}^{\tau}\right)\right)\right)+\sum_{l}\left(C\left(A_{l}^{\tau}\right):: \neg\left(A_{g}^{\tau} \propto A_{l}^{\tau}\right) \wedge \pi\left(A_{l}^{\tau}\right) \geq \pi\left(A_{g}^{\tau}\right)\right. \\
& +\left\{\left(\mathbf{F}_{\tau r \min }+\mathbf{F}_{\tau} \mathrm{n}_{\tau}\right)-\left(M_{\tau} n_{\tau}+m_{\tau}\right)-\mathbf{1}\right\} \bullet\left(\sum _ { l } \left(C\left(A_{l}^{\tau}\right):: \neg\left(A_{i}^{\tau} \propto A_{l}^{\tau}\right) \wedge \pi\left(A_{l}^{\tau}\right) \geq \pi\left(A_{g}^{\tau}\right)\right.\right.
\end{aligned}
$$

\subsubsection{Schedulability Analysis.}

From the above equations, we can calculate the value of $S_{i}^{\tau}(q)$. Once the value of $S_{i}^{\tau}(q)$ is obtained from the above equations, we can iteratively compute $S_{i}^{\tau}(q)$ and $F_{i}^{\tau}(q)$ for $q=1,2,3 \ldots$, 
$42^{\text {nd }}$ IEEE Conference on Decision and Control, Maui, Hawaii, pp. 5932-5938, December 2003

until we reach a $q=m$, such that $F_{i}^{\tau}(q) \leq \mathrm{A}_{\text {rrr }}(m+1)-\mathrm{J}_{\tau}$. Then, the worst-case response time of action $\mathrm{A}_{i}^{\tau}$ is given by:

$$
\mathrm{R}\left(\mathrm{A}_{i}^{\tau}\right)=\max _{q \in[1,2, \ldots, m]}\left\{F_{i}^{\tau}(q)+\mathrm{J}_{\tau}-\operatorname{Arr}_{\tau}(q)\right\}
$$

If the worst-case response time $\mathrm{R}\left(\mathrm{A}_{i}^{\tau}\right)$ is less than or equal to it's deadline $\mathrm{D}\left(\mathrm{A}_{i}^{\tau}\right)$, then the action $\mathrm{A}_{i}^{\tau}$ implementation is feasible. If the worst-case response time $\mathrm{R}\left(\mathrm{A}_{i}^{\tau}\right)$ is larger than the deadline $\mathrm{D}\left(\mathrm{A}_{i}^{\tau}\right)$, then the action $\mathrm{A}_{i}^{\tau}$ implementation is not feasible. If all the action worst-case response times in the real-time control system are less than or equal to their deadlines, we can say that the systems implementation is feasible.

\section{Scheduliability Analysis for the Example System.}

Now, let us revisit our example system and apply the above scheduling analysis method to analyze the system schedulability. Table 2 shows the worst-case response time of each action

\begin{tabular}{|c|c|c|c|c|}
\hline Transaction & Action & Priority & Deadline & Worst Case Response Time \\
\hline \multirow{4}{*}{$\tau_{1}$} & $A_{1}$ & 10 & 300 & 267 \\
\cline { 2 - 5 } & $A_{4}$ & 6 & 800 & 763 \\
\cline { 2 - 5 } & $A_{5}$ & 10 & 300 & 271 \\
\cline { 2 - 5 } & $A_{6}$ & 10 & 280 & 465 \\
\hline \multirow{4}{*}{$\tau_{2}$} & $A_{2}$ & 9 & 460 & 386 \\
\cline { 2 - 5 } & $A_{7}$ & 9 & 400 & 710 \\
\cline { 2 - 5 } & $A_{8}$ & 7 & 720 & 427 \\
\cline { 2 - 5 } & $A_{9}$ & 9 & 450 & 598 \\
\hline \multirow{3}{*}{$\tau_{3}$} & $A_{3}$ & 8 & 620 & 588 \\
\cline { 2 - 5 } & $A_{10}$ & 8 & 600 & 449 \\
\cline { 2 - 5 } & $A_{11}$ & 5 & 480 & 447 \\
\hline
\end{tabular}

Table 2. The Worst Case Response Time for The example systems

which found by this analysis method. From the table, we can see that all the worst-case response time of actions in the system is less than their deadline constraint. So the system is schedulable and feasible. From the table we can also see that the worst case response time of all actions are 
$42^{\text {nd }}$ IEEE Conference on Decision and Control, Maui, Hawaii, pp. 5932-5938, December 2003

large due to action $\mathrm{A}_{11}$ which has large execution time. Since in our system model, the implementation is in uni-processor single thread environments, it causes blocking for all the actions. Based on the table, we can see that the effect of the lower priorities of action $\mathrm{A}_{4}$ and $\mathrm{A}_{8}$ is also reflected in their larger worst case response time because of the greater interference. For non-preemptive scheduling in our uni-processor single thread environments, the worst case response time of the lowest priority action $\mathrm{A}_{11}$ is relatively lower, once the action starts executing, it executes as if its priority is raised to the highest priority in the system.

\section{Conclusion}

Software design has become more and more important within the real-time control system design process since functionality implementation gradually migrated from hardware to software. Consequently, several commercial tools have become available that provide an integrated development environment for real-time control systems with object-oriented techniques to facilitate the design phase. However, these tools lack the "real-time" support required by many of these systems. Especially those with stringent timing constraints.

As a result, we proposed a methodology for the integration of schedilability analysis techniques within UML-RT techniques to support the timing requirements in real-time control system design process. The main contribution of our paper is in the development of the worst case response time analysis for object-oriented design models in which the external events suffer release jitter and have sporadically periodic characteristics, we also extent UML sequence diagram to visually describe the timing properties in real-time control systems. This results developed are also generally applicable to any modeling language using active objects, and explicit communication between objects through message passing. This method can be used to cope with timing constraints in realistic and complex real time control systems. Using this method, a designer can quickly evaluate the impact of various implementation decisions on schedulability. In conjunction 
$42^{\text {nd }}$ IEEE Conference on Decision and Control, Maui, Hawaii, pp. 5932-5938, December 2003

with automatic code-generation, we believe that this will greatly streamline the design and development of real-time control system software.

\section{References}

[1]. G. Booch, J. Rumbaugh, and I. Jacobson. The Unified Modeling Language User Guide. AddisonWesley, 1999.

[2]. J. Rumbaugh, I. Jacobson, and G. Booch. The Unified Modeling Language Reference Manual. Addison-Wesley, 1999.

[3]. B. Selic, G. Gullekson, and P. T. Ward. Real-Time Object-Oriented Modeling. John Wiley and Sons, 1994.

[4]. M. Awad, J. kuusela, and J. Ziegler. Object-Oriented Technology for Real-Time Systems: A Practical Ap-proach using OMT and Fusion. Prentice Hall, 1996.

[5]. B. P. Douglass. Doing Hard Time: Developing Real-Time Systems with Objects, frameworks, and Patterns. Addison-Wesley, 1999.

[6] H. Gomaa. Software Design Methods for Concurrent and Real-Time Systems. Addison-Wesley Publishing Company, 1993.

[7]. A. Burns and A. J. Wellings. HRT-HOOD: A Design Method for Hard Real-Time. Real-Time Systems, 6(1):73-114, 1994.

[8]. L. Kabous and W. Nebel. Modeling hard real-time systems with uml the ooharts approach. In Proceedings, International Conference on Unified Modeling Language (UML'99), 1999.

[9]. K. H. Kim. Object structures for real-time systems and simulators. IEEE Computer, pages 62-70, August 1997.

[10]. Yau, S.S.; Xiaoyong Zhou; Schedulability in model-based software development for distributed real-time systems . Object-Oriented Real-Time Dependable Systems, 2002. (WORDS 2002). Proceedings of the Seventh International Workshop on , 2002 page(s):45-52

[11]. C. Liu and J. Layland. Scheduling algorithm for multiprogramming in a hard real-time environment. Journal of the ACM, 20(1):46-61, January 1973. 
$42^{\text {nd }}$ IEEE Conference on Decision and Control, Maui, Hawaii, pp. 5932-5938, December 2003

[12]. J. Lehoczky, L. Sha, and Y. Ding. The rate monotonic scheduling algorithm: Exact characterization and aver-age case behavior. In Proceedings of IEEE Real-Time Systems Symposium, pages 166-171. IEEE Computer Society Press, December 1989.

[13]. M. Harbour, M. Klein, and J. Lehoczky. Fixed Priority Scheduling of Periodic Tasks with Varying Execution Priority. In Proceedings, IEEE Real-Time Systems Symposium, pages 116-128, December 1991.

[14]. K. Tindell, A. Burns, and A. Wellings. An extendible approach for analysing fixed priority hard realtime tasks. The Journal of Real-Time Systems, 6(2):133-152, March 1994.

[15]. M. Saksena, P. Karvelas. Designing for schedulability: integrating schedulability analysis with object-oriented design. In Real-Time Systems, 2000. Euromicro RTS 2000. 12th Euromicro Conference on , 2000 Page(s): $101-108$

[16]. M. Saksena, P. Karvelas, and Y. Wang. Automatic synthesis of multi-tasking implementations from real-time object-oriented models. In Proceedings, IEEE International Symposium on ObjectOriented Real-Time Distributed Computing, March 2000.

[17]. Paulo Martins, Integrating Real-Time UML Models with Schedulability Analysis. White Papers in Tri-Pacific Software Products 2003 http://www.tripac.com/html/whitepapers/umlsched.pdf

[18]. B. Selic and J. Rumbaugh. Using UML for Modeling Complex Real-Time Systems. White Paper, Published by ObjecTime, and available from www.objectime.com, March 1998.

[19]. Tezuka, T.; Yamashita, T.; Sato, T.; Abiko, Y.; Kanai, T.; Sawada, M. Application of a new automatic gauge control system for the tandem cold mill Industry Applications, IEEE Transactions on , Volume: 38 Issue: 2 , March-April 2002 Page(s): 553 -558.

[20]. J.Y.T.Leung and J. Whitehead. On the complexity of fixed-priority scheduling of periodic, realtimes tasks. Performance Evaluation (Netherlands), 2:237-250, 1982

[21]. M.Joseph and P. Pandya. Finding response times in a real-time systems. Computer Journal (British Computer Society), 29(5):390-395, 1986

[22]. L. Sha, R. Rajkumar, and J. Lehocaky. Priority inheritance protocols: An approach to real-time synchronization. IEEE Transactions on Computers, 39:1175-1185, September 1990. 
$42^{\text {nd }}$ IEEE Conference on Decision and Control, Maui, Hawaii, pp. 5932-5938, December 2003

[23]. J.P.Lehoczky. Fixed priority scheduling of periodic task sets with arbitrary deadlines. In Proceedings of IEEE Real-Time Systems Symposium, pages 201-209. IEEE Computer Society Press, December 1990.

[24]. A. Bertossi and A. Fusiello. Rate-monotonic scheduling for hard-real-time systems. European Journal of Operational Research, pages 429-443,, June 1996

[25]. C. J. Fidge. Real-time schedulability tests for preemptive multitasking. The Journal of Real-Time Systems, Pages 61-93, 1998. 